\title{
Limitations of Immunohistochemistry in Diagnosis of a Primary Mucinous Carcinoma of the Skin and Its Metastasis
}

\author{
Mann $\mathrm{C}^{1^{*}}$, Schad $\mathrm{A}^{2}$, Mann $\mathrm{W}^{3}$, Weidenthaler-Barth $\mathrm{B}^{1}$ \\ ${ }^{1}$ Department of Dermatology, Johannes Gutenberg University Mainz, Germany \\ ${ }^{2}$ Department of Pathology, Johannes Gutenberg University Mainz, Germany \\ ${ }^{3}$ Department of Otorhinolaryngology, Roemerwall Clinic Mainz, Germany
}

Corresponding Author: Caroline Mann, MD

Address: Department of Dermatology, University Medical Centre, 55131 Mainz, Germany.

Received date: 24 November 2020; Accepted date: 21 December 2020; Published date: 29 December 2020

Citation: Mann C, Schad A, Mann W, Weidenthaler-Barth B. Limitations of Immunohistochemistry in Diagnosis of a Primary Mucinous Carcinoma of the Skin and Its Metastasis. Asp Biomed Clin Case Rep. 2020 Dec 29;4(1): $11-15$.

Copyright (C) 2021 Mann C, Schad A, Mann W, Weidenthaler-Barth B. This is an open-access article distributed under the Creative Commons Attribution License, which permits unrestricted use, distribution, and reproduction in any medium provided the original work is properly cited.

\begin{abstract}
We describe the case of a 67-year-old female patient who presented with an unclear swelling on the right supraclavicular neck, two years after resection of a mucinous carcinoma on the right mastoid. Two pathological reports from separate universities diagnosed the primary mastoid skin lesion as a metastatic adenocarcinoma. Strikingly, GATA binding protein 3 (GATA3) and mammaglobin, both immunomarkers found in breast cancer, were positive. An urgent search for the primary tumor most likely in the breast was commenced. However, as no other primary tumor could be detected at that time, a Primary Mucinous Carcinoma of the Skin (PMCS) was also debated.

Two years later neck node enlargement was suspicious for lymph node metastasis during ultrasound examination and conservative neck dissection was performed. Immunohistochemistry revealed again GATA 3, Mammaglobin, estrogen, and progesterone receptor positive tissue. Using a variety of other markers, we were unable to detect neither significant morphological nor immunohistochemical characteristics that distinguished the lesion from a mucinous carcinoma of mammary origin. Following a detailed review of the clinical context, we concluded the lesion to be consistent with a late metastasis of a PMCS. This report demonstrates the limitations of currently used histopathological and immunohistochemical differentiation in metastatic mucinous carcinoma.
\end{abstract}

\section{Keywords}

Immunohistochemistry, Carcinoma, Diagnosis, Immunostaining

\section{Introduction}

PMCS is a rare tumor that infrequently metastasizes but has a high local recurrence rate [1]. The reason for its rare metastatic potential is seen in the poor vascularization [2]. PMCS was first described by Lennox et al in 1952 [3]. Since then only a few case reports and other isolated reports about metastatic disease exist. 
Clinically the tumor presents as a solitary greyish, red, and purple skin lesion with telangiectasia [1]. Histologically a circumscribed tumor with lakes of mucin, neoplastic cells arranged in strands and nests, and apocrine secretion is frequently observed (Fig-1) [4].

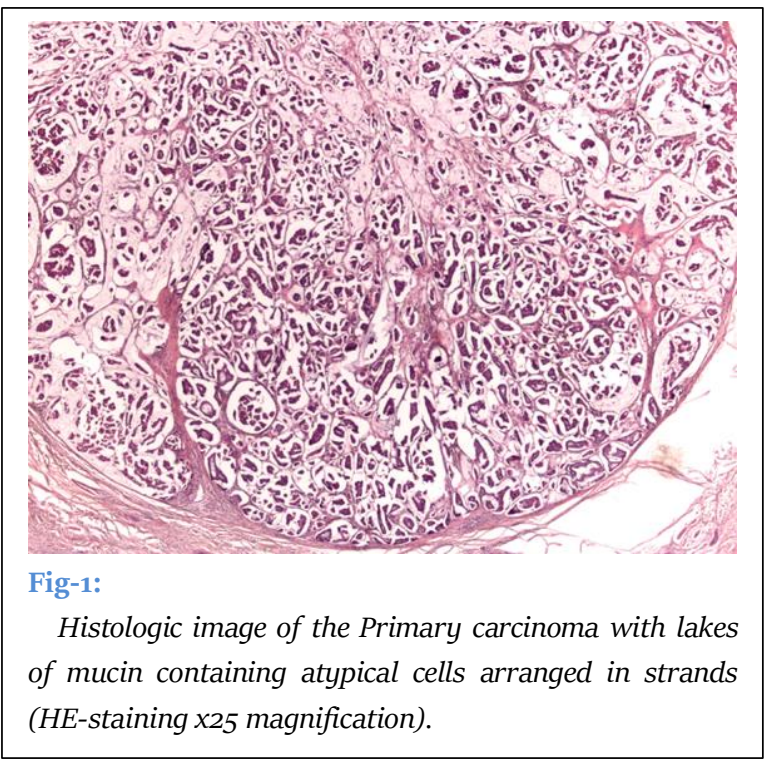

The literature repeatedly describes difficulties in diagnosing PMCS, especially when attempting to differentiate the metastases from mucinous carcinomas of other origins. Therefore, various additional immunomarkers are being discussed. Clinically the localization of the lesion can be indicative. Mucinous skin carcinomas are mostly seen in the head and neck region and isolated specimens from this area should raise suspicion for a PMCS. In contrast, metastases located in the axillary region are more likely to be related to a primary tumor in the breast while metastases on the lower trunk are mostly seen in colorectal carcinomas.

\section{Case Report}

A 67-year-old female presented with a suspicious swelling on the right supraclavicular region two years after resection of a mucinous carcinoma on the surface of the skin overlying the right mastoid. The mastoid skin lesion was originally diagnosed by two separate institutes to be most likely a metastatic mucinous carcinoma. Approaches to better define the lesion in regards of a potential primary skin origin were made using smooth muscle markers like smooth muscle actin, p63, and D2-40, however, no positivity was found.

Because of positivity for GATA 3 ( an immunomarker found for example in breast and salivary glands but also in skin tumors $[5,6]$ and expression of mammaglobin, an immunohistochemical marker found in breast cancer [7], mammography was done also and ultrasonography was performed to rule out a salivary gland origin because of its anatomic proximity. Moreover, colonoscopy was performed because of positive staining for mucin (MUC) 2 and 6 , as well as cytokeratin 7 ( $\left.\mathrm{CK}_{7}\right)$. However, no primary was detected. Two years later because of a swelling in the right lower neck, a CT and MRI scan were performed and interpreted as an atheroma-like lesion in level $\mathrm{V}$ of the right neck with a recommendation for extirpation.

Additional PET-CT investigation revealed no further lesions but considered this lesion to be of inflammatory origin. Ultrasonography, at last, revealed a suspected lymph node metastasis of unknown primary and a limited dissection of level IV and V of the right neck followed. Morphologic and immunohistochemical findings were indicative of a papillary, partially mucinous differentiated metastasis. This raised again suspicion of a primary breast carcinoma following immunohistochemical characteristics: a positivity for pan-cytokeratin and cytokeratin 7, progesterone receptor (PR), and estrogen receptor, especially positivity for estrogen receptor (ER), and GATA-3. However, the lesion was negative for caudal-related homeobox gene 2 (CDX-2), a marker for gastrointestinal tumors. As a repeated mammogram and also a repeated colonoscopy were again negative, the diagnosis of a late metastasis of a PMCS was made. Due to extracapsular growth of the late lymph node-metastasis, the mastoid as the site of the primary and the right neck was radiated to 60 gray.

\section{Discussion}

The patient described in this report was confronted twice with the diagnosis of mucinous carcinoma. Each time elaborate and costly diagnostics were required to rule out metastatic disease and to initiate adequate therapy. The imaging technology was of little help 
Citation: Mann C, Schad A, Mann W, Weidenthaler-Barth B. Limitations of Immunohistochemistry in Diagnosis of a Primary Mucinous Carcinoma of the Skin and Its Metastasis. Asp Biomed Clin Case Rep. 2020 Dec 29;4(1): 11-15.

except for ultrasound to identify a metastatic lymph node involvement. In this case, PET-CT was not sensitive enough to define this PMCS metastasis as malignant and as the lesion was suspected to be an atheroma. On ultrasonography, the lesion met the criteria for possible malignancy. The gold standard for a diagnosis is histology and immunohistochemistry but both reached their limitations.

Differentiation of PMCS from metastatic mucinous breast carcinoma (MMBC) and other mucinous cancer metastasis is known to be challenging (Table-1). This is further complicated in the case of lymph node metastasis as in this case. Establishing morphologic criteria to help distinguish between PMCS and mucinous metastasis of a primary tumor (e.g. of the breast or GI-tract) proved to be challenging. The clinical experience that most metastases of the GI tract tumors occur on the trunk and are unlikely to present in the head and neck region helped to rule out this differential diagnosis. Because of the location of the metastasis in the right lower neck, a primary breast tumor was more likely.

Table-1: Expression of immunomarkers in primary mucinous carcinomas of the skin (PMCS), mucinous metastasis of the colorectal tract and the mucinous Mamma carcinomas [8,10-12]

\begin{tabular}{|l|l|l|}
\hline PMCS & GI MA & Mamma Carcinoma (MMBC) \\
\hline CK7 +, CK20- & CK7+, CK20+ & CK7,+ CK20- \\
\hline MUC2, MUC6 & MUC 6, MUC 2 & MUC 1,2,6 \\
\hline ER, PR & & ER, PR \\
\hline CDX2 Negative & CDX2 Positive & CDX2 Negative \\
\hline Mammaglobin & & Mammaglobin \\
\hline GATA 3 positive & & GATA 3 Positive \\
\hline & Beta Catenin & Beta Catenin \\
\hline
\end{tabular}

In this patient mammaglobin, a marker frequently used in breast cancer was positive in the extirpated lymph node metastasis, plus endocrine differentiation with ER and PR were also seen. HER-2 molecular staining in concordance with literature reports was negative [8].

Qureshi et al. [9] determined that in order to differentiate a primary mucinous skin tumor from breast tumor metastasis detection of in situ components with myoepithelial cells may be helpful, better demonstrated by smooth muscle actin or p63 $[10,11]$.

Positivity would have favored the diagnose of PMCS and militate against a mucinous metastasis of another cancerous origin. Staining of the primary was performed using SMA and p63, however, no positivity was seen.
The difficulty of distinguishing between tumor origins was increased when confronted with the lymph node metastasis. We found GATA-3 expression in the primary skin tumor lesion in this patient and also in the metastasis (Fig-2). However, other studies have already demonstrated a lack of specificity of this marker as it can also be detected in MMBC [12]

The absence of CK 20 could help to exclude metastatic disease of the colon as well as $\mathrm{CK}_{5} / 6$. Because of the location over the mastoid, a salivary gland tumor was excluded also using ultrasonography. The expression of different mucin positive proteins was not specific enough, since studies have shown that they can be expressed in various mucinous carcinomas [12]. However, CDX-2 belonging to the homeobox protein family, and beta-catenin (a cell adhesion molecule), are mostly positive in mucinous carcinomas of the GI tract, but negative in both PMCS and MMBC [13]. Mucinous carcinoma is a lesion that rarely metastasizes but has a high local recurrence rate. Once 
Citation: Mann C, Schad A, Mann W, Weidenthaler-Barth B. Limitations of Immunohistochemistry in Diagnosis of a Primary Mucinous Carcinoma of the Skin and Its Metastasis. Asp Biomed Clin Case Rep. 2020 Dec 29;4(1): 11-15.

it does metastasize, the prognosis becomes poorer $[1,2]$. So far it is not possible to make a clear statement about the prognosis in this case report, as there are almost no reports on metastatic mucinous carcinomas and especially in late metastasizing tumors. Due to the different treatment modalities, a definite distinction of metastatic PMCS from other mucinous metastasis is indispensable. Because of its metastatic potential close follow-up is advised. In the end, only its location, as well as the same histologic characteristics, favored the diagnosis of a lymph node metastasis of a PMCS.

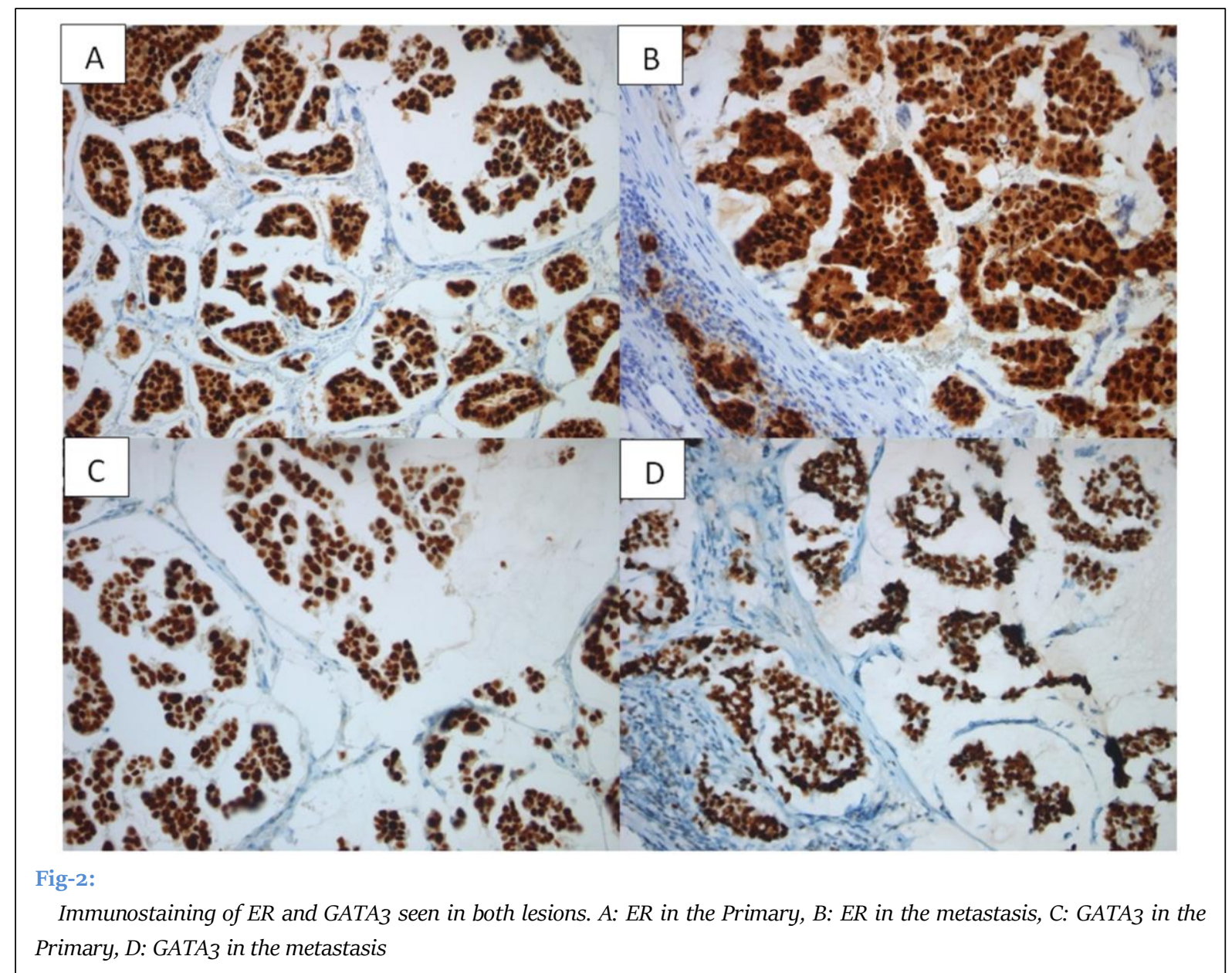

\section{Acknowledgments}

We are grateful to Dr. Ronald Amedee and Mrs. Ulrike Rady-Pizzaro for their help in drafting the manuscript.

\section{Funding Information}

This report received no specific grant from any funding agency in the public, commercial, or not-forprofit sectors.

\section{Conflict of Interests}

All authors have read and approved the final version of the manuscript. The authors have no conflicts of interest to declare.

\section{References}

[1] Maerki J, Ahmed S, Lee E. Primary mucinous carcinoma of the skin. Eplasty. 2013 Jun 18;13:ic47. [PMID: 23814638]

[2] Scilletta A, Soma PF, Grasso G, Scilletta R, Pompili G, Tarico MS, Perrotta RE. Primary cutaneous mucinous carcinoma of the cheek. Case report. G Chir. 2011 Jun-Jul;32(6-7):323-25. [PMID: 21771401]

[3] Lennox B, Pearse AG, Richards HG. Mucinsecreting tumours of the skin with special reference to the so-called mixed-salivary tumour of the skin and its relation to hidradenoma. J Pathol Bacteriol. 1952 Oct;64(4):865-80. [PMID: 13000598]

[4] Elder DE, Massi D, Scolyer RA, Willemze R, editors. WHO Classification of Skin Tumours. 4th ed. Lyon, 
France: IARC; 2018:72-75.

[5] Miettinen M, McCue PA, Sarlomo-Rikala M, Rys J, Czapiewski P, Wazny K, Langfort R, Waloszczyk P, Biernat W, Lasota J, Wang Z. GATA3: a multispecific but potentially useful marker in surgical pathology: a systematic analysis of 2500 epithelial and nonepithelial tumors. Am J Surg Pathol. 2014 Jan;38(1):13-22. [PMID: 24145643]

[6] Wong FK, Zumsteg ZS, Langevin CJ, Ali N, Maclary S, Balzer BL, Ho AS. Mucinous Carcinoma with Neuroendocrine Differentiation of Salivary Gland Origin. Head Neck Pathol. 2017 Jun;11(2):249-55. [PMID: 27534564]

[7] Wang Z, Spaulding B, Sienko A, Liang Y, Li H, Nielsen G, Yub Gong G, Ro JY, Jim Zhai Q. Mammaglobin, a valuable diagnostic marker for metastatic breast carcinoma. Int J Clin Exp Pathol. 2009;2(4):384-89. Epub 2008 Dec 1. [PMID: 19158935]

[8] Kazakov DV, Suster S, LeBoit PE, Calonje E, Bisceglia M, Kutzner H, Rütten A, Mentzel T, Schaller J, Zelger B, Baltaci M, Leivo I, Rose C, Fukunaga M, Simpson RH, Yang Y, Carlson JA, Cavazza A, Hes O, Mukensnabl P, Vanecek T, Fidalgo A, Pizinger K, Michal M. Mucinous carcinoma of the skin, primary, and secondary: a clinicopathologic study of 63 cases with emphasis on the morphologic spectrum of primary cutaneous forms: homologies with mucinous lesions in the breast. Am J Surg Pathol. 2005 Jun;29(6):764-82. [PMID: 15897743]

[9] Qureshi HS, Salama ME, Chitale D, Bansal I, Ma CK, Raju U, Ormsby A, Lee MW. Primary cutaneous mucinous carcinoma: presence of myoepithelial cells as a clue to the cutaneous origin. Am J Dermatopathol. 2004 Oct;26(5):353-58. [PMID: 15365364]

[10] Albasri AM, Ansari IA, Aljohani AR, Alhujaily AS. Primary mucinous adenocarcinoma of the eyelid: A case report and literature review. Saudi Med J. 2018 Sep;39(9):940-45. [PMID: 30251739]

[11] Mardi K, Diwana VK. Primary cutaneous mucinous carcinoma: a rare entity. Indian Dermatol Online J. 2011 Jul;2(2):82-84. [PMID: 23130231]

[12] Mertens RB, de Peralta-Venturina MN, Balzer BL, Frishberg DP. GATA3 Expression in Normal Skin and in Benign and Malignant Epidermal and Cutaneous Adnexal Neoplasms. Am J Dermatopathol. 2015 Dec;37(12):885-91. [PMID: 26595821]

[13] García-Labastida L, Garza-Guajardo R, BarbozaQuintana O, Rodríguez-Sanchez IP, Ancer-Rodríguez J, Flores-Gutierrez JP, Gómez-Macías GS. CDX-2, MUC-2 and B-catenin as intestinal markers in pure mucinous carcinoma of the breast. Biol Res. 2014 Sep 18;47(1):43. [PMID: 25299496] 